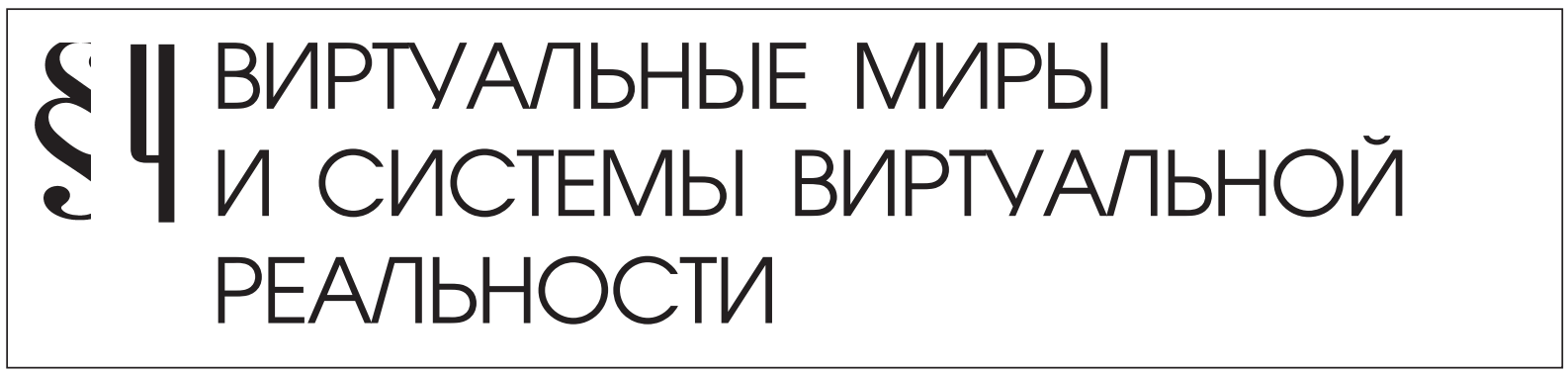

Сморкалов А.Ю., Садовин И.А.

\title{
СИСТЕМА ПЕРЕНОСА ЛЕКЦИЙ В ВИРТУАЛЬНЫЙ МИР vAcademia С ИСПОЛЬЗОВАНИЕМ ВОЗМОЖНОСТЕЙ Microsoft Kinect И ПОТОКОВЫХ ПРОЦЕССОРОВ
}

Аннотация: В данной статье предлагаются методы и архитектура системы переноса «живых» лекций в виртуальный мир vAсаdетіа. Система записывает голос лектора и использует Microsoft Kinect для захвата жестов преподавателя, преобразуя эти данные в анимацию аватара лектора. На основе использования возможностей потоковых процессоров содержимое экрана проектора, который использует преподаватель, переносится непосредственно в виртуальный мир и отображается в совместном графическом рабочем пространстве, а также происходит дополнительный анализ изображения с видеокамеры Kinесt для более точного определения положения тела преподавателя. Использование потоковых процессоров позволило эффрективно реализовать передачу содержимого экрана проектора на совместое рабочее пространство в виртуальном мире, а также осуществлять распознавание иявтных маркеров на теле преподавателя практически без потери производительности. Применение Kinect для захвата лекичй (совместно с получением изображения с проектора или интерактивной доски и отображением его на виртуальной доске) могло бы позволить учителям использовать виртуальные миры как мощное средство обучения, находясь в привычной для себя среде обучения. Это и является иелью данной работы. Ключевые слова: vAcademia, Microsoft Kinect, nотоковые процессоры, анализ изображения, виртуальный мир, захват лекций, презентация, слайдовые лекции, захват движения, дискретные вейвлет-преобразования 


\section{1. Введение}

Виртуальные миры применяются для обучения в сотнях университетов по всему миру ${ }^{1}$. Их главным недостатком для учителей является сложность перехода от преподавания в реальной аудитории к организации занятий в виртуальном мире ${ }^{2}$.

Microsoft Kinect - недорогое устройство для захвата движений человека ${ }^{3}$ Устройство включает в себя видеокамеру, камеру глубины и IK-камеру. Для управления устройством и получения данных с него Microsoft бесплатно предоставляет Kinect SDK.

Применение Kinect для захвата лекций (совместно с получением изображения ектора или интерактивной доски и отображением его на виртуальной доске) могло бы позволить учителям использовать виртуальные миры как мощное средство обучения, находясь в привычной для себя среде обучения. Это и является целью данной работы.

\section{2. Обзор способов записи лекций}

Наиболее простой способ записи лекций - это запись лекций в форме видео. В этом случае требуется участие видеооператора, а при просмотре теряется эффект присутствия.

Другой популярный способ записи лекций - это вебинары. Он не требует участия ассистента для введения записи. Однако с одной стороны вебинары не являются привычной средой обучения для типичного преподавателя и ученика, а с другой стороны в занятии в вебинаре также отсутствует эффект присутствия.

Виртуальная академия (vAcademia) - единственная в мире образовательная трехмерная виртуальная среда с реализованной возможностью 3D-записи занятий, не отличающихся от оригинала. 3D-запись позволяет захватить все, что в данный момент находится в виртуальном мире, в том числе положение объектов, внешний вид и движения аватаров, содержимое интерактивной доски, текст и сообщения голосового чата.

3D-запись концептуально отличается от записи видео или захвата экрана. 3D-запись содержит всю 3D-сцену со всеми 3D-объектами и аватарами. Все действия аватаров также сохраняются в 3D-записи. Эти действия повторятся, когда 3D-запись будет воспроизведена. Её может посетить группа студентов, которые могут взаимодействовать друг с другом и с записанными объектами. Хотя 3D-запись дает преподавателю уникальные возможности, а ученикам эффект присутствия, использование vAcademia затрудняется

\footnotetext{
1 Сморкалов, А.Ю. Реализация образовательных инструментов в виртуальных 3D-средах с использованием потоковых процессоров / А.Ю. Сморкалов // Образовательные технологии и общество. - Т. 14. № 3, 2011. - С. 409-425. 2 Mikhail Morozov, Alexey Gerasimov, Mikhail Fominykh, Andrey Smorkalov. Asynchronous Immersive Classes in a 3D Virtual World: Extended Description of vAcademia // Trans. on Comput. Sci. XVIII, LNCS 7848, p. 81-100.

3 Microsoft, Kinect. Web: http://www.microsoft.com/en-us/kinectforwindows.
} 
необходимостью использования непривычной для учителей среды. Разработка системы, позволяющей получать 3D-записи при обучении преподавателем в привычной среде обучения, является целью данной работы.

\section{3. Первый прототип}

Интерфейс режима виртуализации «живых» лекций реализован с использованием скриптов vAcademia. Скрипты инициируют начало и конец этого режима, осуществляют отображение информации, включающей скелет в 2D и статус распознавания каждой части тела, включают и выключают режим передачи динамического изображения с экрана проектора на виртуальную доску. Передача указанного динамического изображения осуществляется на основе использования для сжатия данных дискретного вейвлет преобразования, реализованного с использованием вычислительных возможностей потоковых процессоров ${ }^{4}$.

Скрипты взаимодействуют с библиотекой поддержки анимированных объектов (Animation library) графического движка через Script Executing Library. Animation library взаимодействует с плагином поддержки Kinect, получая от него набор ключевых точек скелета захватываемого человека и ориентируя на основе этих данных скелет аватара преподавателя (рис. 1). B vAcademia для реализации скелетной анимации аватаров ис-

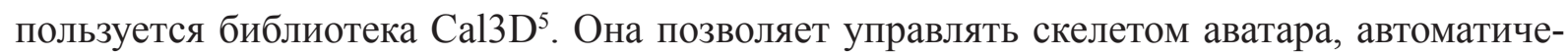
ски пересчитывая его полигональную 3D-модель под новое состояние скелета. Поддерживается режим «преподаватель сидит», в котором по данным Kinect ориентируются только руки и голова.

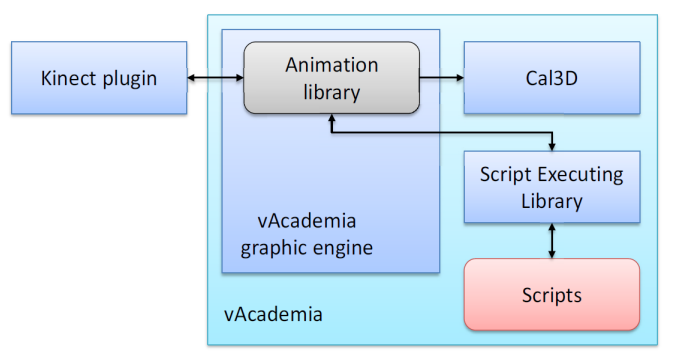

Рис. 1. Архитектура подсистемы захвата движений лектора

4 Сморкалов, А.Ю. Математическая и программная модели генерации текстур на графических потоковых процессорах / А.Ю. Сморкалов // Программные системы и вычислительные методы. - № 1, 2013. - С. 116-128.

5 L. Jingtang, Z. Gang, T. Dunming, and X. Junjie "Research of skeletal-based virtual human animation using Cal3D," in 3rd International Conference on System Science, Engineering Design and Manufacturing Informatization (ICSEM) Chengdu, China, 2012, p. 269-273. 


\section{Система переноса лекций в виртуальный мир vAcademia}

Kinect возвращает статус каждой точки скелета (не распознана; распознана предположительно; распознана точно) и информацию о том, не обрезан ли скелет по одному из четырех направлений областью видимости камеры. Было выделено 5 основных частей скелета: руки, ноги, голова. Качество захвата каждой из частей через Kinect оценивается отдельно. Если часть скелета перестает адекватно распознаваться, она перестает управляться на основе данных Kinect и остается спозиционированной по последним полученным корректным точкам. Если через некоторый небольшой промежуток времени часть тела не начинает распознаваться вновь, часть тела возвращается в положение по умолчанию.

Первые версии прототипа были слишком неустойчивы для нормального применения в учебном процессе, и использовались чисто в качестве эксперимента. По итогам тестирования прототипа был выявлен ряд недостатков и были сформированы предложения по его улучшению.

\section{4. Применение механизма захвата движения Kinect в vAcademia}

\section{1. Проблемы}

Первоначальная реализация выявила два главных недостатка Kinect:

1. Недостаточная точность захвата движений. Kinect SDK не возвращает точки идеально движущегося скелета человека, вместо этого предоставляется возможность управлять коэффициентами усреднения координат, предсказания движения, получать данные о корректности точки скелета и т.д. Несмотря на использование всей рассмотренной ранее информации, не удалось построить надежную модель аватара, который будет двигаться без неестественных поз. Между тем, при передаче движений преподавателя искажения и неествественные позы недопустимы.

2. Kinect не распознает повороты аватара, левая рука всегда располагается слева, правая - справа. Если захватываемый человек отворачивается от устройства на 180 градусов, Kinect путает правую и левую половину тела, возвращая совершенно неествественную позу.

\section{2. Предложенные решения описанных проблем}

1. Для решения проблем неестественных поз было принято решение ограничить расстояние от устройства Kinect до аватара, на котором работает распознавание. Экспериментальным путем было выяснено, что скелет человека начинает распознаваться без существенных искажений начиная с 1,8 м (с 1,3 м - в сидячем режиме). Кроме того, было 
решено зафиксировать положение Kinect на расстоянии 0,5 метра от пола и программно поворачивать устройство в 0 градусов по вертикальной оси, чтобы захватываемый человек оказывался в строго перпендикулярной плоскости.

Кроме того, была введена фильтрация неестественных положений частей тела. Для этого было реализовано ограничение допустимых значений эйлеровых углов между костями и, кроме того, в отдельную часть тела были выделены кисти, чтобы оценивать естественность их положения отдельно. В случае, если проверка на естественное положение имеет отрицательный результат, часть тела считается не распознанной.

2. Kinect не определяет правильный поворот человека, несмотря на то, что есть возможность получить кватернион поворота всего тела - получаемый поворот далек от реальности. Между тем, возможность частичного поворота очень важна при проведении лекции преподавателем. Поэтому был реализован следующий алгоритм:

- Поворот определяется линейной функцией от положения крайних точек таза. Однако с учетом принципов работы Kinect, его значение корректно в пределах примерно $(-110,110)$ градусов относительно положения «лицом к Kinect». При более сильном повороте, работа алгоритма сбивается, и рассчитанный поворот не может быть признан корректным.

- Были введены цветные маркеры (2 шт.), которые располагаются на туловище, справа/спереди и слева/спереди (рис. 2). На практике используются маркеры красного и зеленого цветов. Видеопоток, получаемый от устройства Kinect, aнализируется с использованием потоковых процессоров. Модель, описанная в работе $^{6}$, была расширена понятием функции-трафарета спроецированной фигуры:

$$
B_{R}(x, y)=\left\{\begin{array}{l}
0, \text { если } G_{R}(x, y)=\phi \\
1, \text { если } G_{R}(x, y) \neq \phi
\end{array}\right.
$$

С помощью (1) была задана функция количества пикселей спроецированной фигуры $\mathrm{S}_{\mathrm{P}}$ на растеризованном изображении:

$$
K_{\eta}\left(\mathcal{S}_{p}\right)=\sum_{x=1}^{W} \sum_{y=1}^{H} B_{k}(x, y)
$$

Изображение обрабатывается фильтром, который использует $\phi$ как цвет точки, если точка не соответствует цвету маркера с заданной погрешностью. Если значение

\footnotetext{
6 Сморкалов, А.Ю. Математическая и программная модели генерации текстур на графических потоковых процессорах / А.Ю. Сморкалов // Программные системы и вычислительные методы. - № 1, 2013. - С. 116-128.
} 
функции (2) больше заданного значения, то считается, что маркеры распознаны и аватар находится в допустимом диапазоне поворота, если нет, то прекращается использование данных Kinect до возобновления распознавания маркеров.

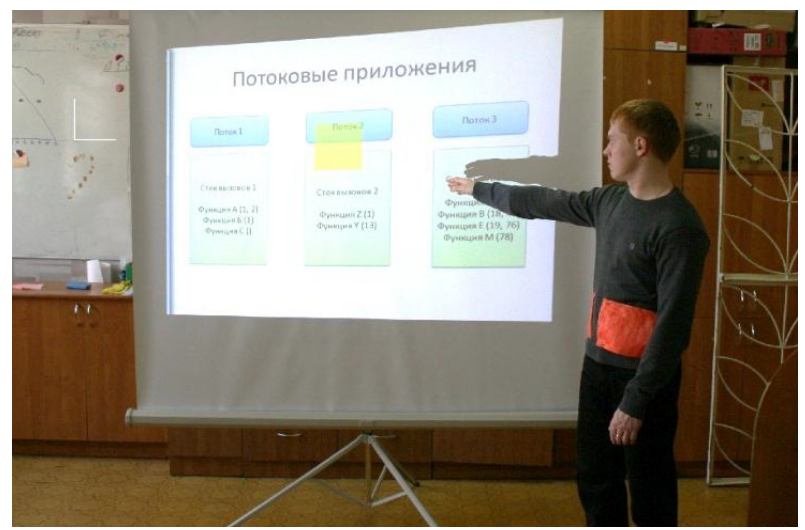

Рис. 2. Процесс захвата лекции

Kinect возвращает для каждой точки изображения число, говорящее о том, к какому из распознанных людей он относит эту точку. Эта информация используется для того, чтобы выполнять распознавание маркеров только на теле человека, игнорируя фон.

\section{5. Инструменты поддержки слайдовых лекций}

\section{1. Проблемы}

При проведении слайдовых лекций с устройством Kinect были выявлены три следующие проблемы:

1. Перемещение и положение преподавателя относительно реальной доски и виртуальной должны совпадать.

2. Есть необходимость использовать указку, но Kinect не может её захватить.

3. Есть необходимость перелистывать слайды, однако это требует взаимодействия с компьютером, что нарушает концепцию передачи занятия в виртуальность, где эти действия преподавателя не будут восприняты адекватно.

\section{2. Предложения и решения}

1. Настройка Kinect должна производиться каждый раз после перемещения экрана проектора или самого устройства Kinect. В режиме настройки преподаватель отмечает левой и правой рукой соответственно правый и левый края экрана, которые захватываются в координатах Kinect. C помощью этого достигается соответствие между пере- 
мещением преподавателя у экрана проектора и перемещением аватара преподавателя у виртуальной доски. Виртуальная доска размещается в виртуальном мире с размерами, совпадающими с размерами экрана проектора. Так достигается соответствие как по длине доски, так и по её высоте.

2. Указка по направлению руки. Если луч на продолжении руки аватара пересекает поверхность виртуальной доски, то от пальцев руки к точке на доске, на которую показывает преподаватель, в виртуальном мире появляется указка (рис. 3).

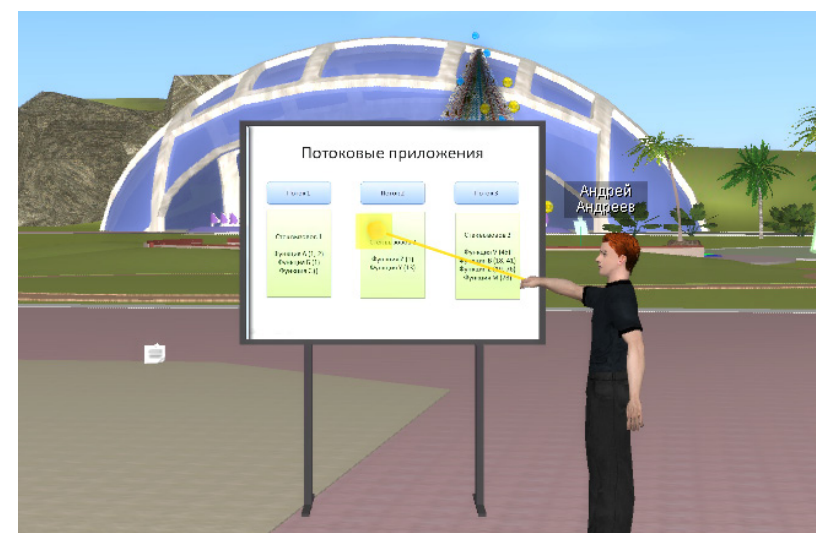

Рис. 3. Кадр из 3D-записи лекции, полученной с использованием Kinect

Для того, чтобы показать преподавателю, на какую область доски указывает его рука, на доске на конце указки показывается полупрозрачный желтый квадрат (рис. 2). Таким образом, преподаватель знает, куда направлена виртуальная указка своего аватара в любой момент времени (рис. 3).

3. Перелистывание слайдов в PowerPoint происходит с помощью распознавания жестов Swipe Left и Swipe Right на основе отслеживания контрольных точек скелета аватара на конце обоих рук.

\section{6. Выводы}

Были предложены архитектура системы переноса «живых» лекций в виртуальный мир vAcademia, а также методы решения нескольких важных проблем, связанных с захватом движений преподавателя, таких как, например, проблема некорректной передачи положения скелета преподавателя и отслеживания поворота тела. Были реализованы инструменты проведения лекции на основе презентации с использованием Kinect.

Использование потоковых процессоров позволило эффективно реализовать передачу 
содержимого экрана проектора на совместое рабочее пространство в виртуальном мире, а также осуществлять распознавание цветных маркеров на теле преподавателя практически без потери производительности. Подробное сравнение эффективности реализации дискретных вейвлет-преобразований для сжатия динамических изображения на потоковых процессорах и на центральном процессоре приведено в работе ${ }^{7}$ Замеры влияния включения распознавания цветных маркеров на потоковых процессоров на общую производительность vAcademia, усредненные по 20 различным аппаратным конфигурациям, показали, что производительность уменьшается на 0,8\%, что является приемлимым результатом.

\section{Библиография:}

1. Сморкалов, А.Ю. Реализация образовательных инструментов в виртуальных 3D-средах с использованием потоковых процессоров / А.Ю. Сморкалов // Образовательные технологии и общество.-Т. 14. № 3, 2011.-С. 409-425.

2. Сморкалов, А.Ю. Математическая и программная модели генерации текстур на графических потоковых процессорах / А.Ю. Сморкалов // Программные системы и вычислительные методы.-№ 1, 2013.-С. 116-128.

DOI: $10.7256 / 2305-6061.2013 .01 .10$

3. L. Jingtang, Z. Gang, T. Dunming, and X. Junjie "Research of skeletal-based virtual human animation using Cal3D," in 3rd International Conference on System Science, Engineering Design and Manufacturing Informatization (ICSEM) Chengdu, China, 2012, p. 269-273. 4. Mikhail Morozov, Alexey Gerasimov, Mikhail Fominykh, Andrey Smorkalov.

Asynchronous Immersive Classes in a 3D Virtual World: Extended Description of vAcademia // Trans. on Comput. Sci. XVIII, LNCS 7848, p. 81-100.

5. Microsoft, Kinect. Web: http://www.microsoft.com/en-us/kinectforwindows.

\section{References:}

1. Smorkalov, A.Yu. Realizatsiya obrazovatel'nykh instrumentov v virtual'nykh 3D-sredakh s ispol'zovaniem potokovykh protsessorov / A.Yu. Smorkalov // Obrazovatel'nye tekhnologii i obshchestvo.-T. 14. № 3, 2011.-S. 409-425.

2. Smorkalov, A.Yu. Matematicheskaya i programmnaya modeli generatsii tekstur na graficheskikh potokovykh protsessorakh / A.Yu. Smorkalov // Programmnye sistemy i vychislitel'nye metody.-№ 1, 2013.-S. 116-128.

DOI: $10.7256 / 2305-6061.2013 .01 .10$

3. L. Jingtang, Z. Gang, T. Dunming, and X. Junjie "Research of skeletal-based virtual human animation using Cal3D," in 3rd International Conference on System Science, Engineering Design and Manufacturing Informatization (ICSEM) Chengdu, China, 2012, p. 269-273.

7 Сморкалов, А.Ю. Математическая и программная модели генерации текстур на графических потоковых процессорах / А.Ю. Сморкалов // Программные системы и вычислительные методы. - № 1, 2013. - С. 116-128. 
Программные системы и вычислительные методы - №4(5) • 2013

4. Mikhail Morozov, Alexey Gerasimov, Mikhail Fominykh, Andrey Smorkalov. Asynchronous Immersive Classes in a 3D Virtual World: Extended Description of vAcademia // Trans. on Comput. Sci. XVIII, LNCS 7848, p. 81-100.

5. Microsoft, Kinect. Web: http://www.microsoft.com/en-us/kinectforwindows. 